Supporting Information for

\title{
High-Sensitivity and High-Efficiency Detection of DNA Hydroxymethylation in Genomic DNA by Multiplexing Electrochemical Biosensing
}

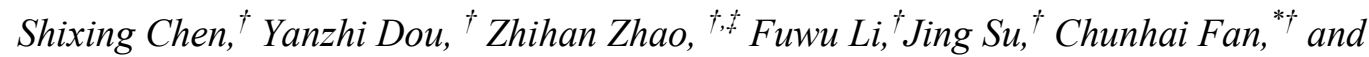
Shiping Song ${ }^{* \dagger}$

$\dagger$ Division of Physical Biology \& Bioimaging Centre, Shanghai Synchrotron Radiation Facility, CAS Key Laboratory of Interfacial Physics and Technology, Shanghai Institute of Applied Physics, Chinese Academy of Sciences, Shanghai 201800, China

${ }^{\ddagger}$ College of Sciences, Shanghai University, Shanghai 200444, China

*to whom correspondence should be addressed. E-mail: spsong@sinap.ac.cn.

This file includes:

Template DNA and modified complementary DNA sequences 


\section{Template DNA and modified complementary DNA sequences}

Template DNA ,

5' ATA GAA ATT GCA TCA ACG CAT ATA GCG CTA GCA GCA CGC CAT AGT GAC TGG CGA TGC TGT-3'.

Complementary DNA, ACA GCA TCG CCA GTC ACT ATG GCG TGC TGC TAG CGC TAT ATG CGT TGA TGC AAT TTC TAT-3'.

Complementary DNA containing 5-hmC,

A $\underline{C}^{\text {hm }}$ A GCA TCG CCA GTC ACT ATG GCG TGC TGC TAG CGC TAT ATG CGT TGA TGC AAT TTC TAT-3'.

Complementary DNA containing 5-mC, A $\underline{C}^{\mathrm{m}}$ A GCA TCG CCA GTC ACT ATG GCG TGC TGC TAG CGC TAT ATG CGT TGA TGC AAT TTC TAT-3'.

Complementary DNA containing 5-fmC, A $\underline{c}^{\mathrm{fm}}$ A GCA TCG CCA GTC ACT ATG GCG TGC TGC TAG CGC TAT ATG CGT TGA TGC AAT TTC TAT-3'. 